\title{
Percepción del equipo de salud sobre los beneficios del calostro como factor protector de enterocolitis necrotizante en recién nacidos prematuros
}

\author{
Health professional's perception about the use of human colostrum, as preventive \\ measure for necrotizing enterocolitis in preterm newborns
}

\author{
Juan Pablo Rojas Beytía $a^{\mathrm{a}, \mathrm{b}}$, José Cariaga Irarrázabal ${ }^{c}$, Francisca Castro Guerrero ${ }^{c}$, Paula Domingo Carrasco ${ }^{c}$, \\ Keila Fernández Pérez ${ }^{\mathfrak{c}}$, Ivette Pavez Ortiz ${ }^{\mathrm{c}}$, Nicole Ghislaine Iturrieta Guaita ${ }^{\mathrm{d}}$, Ana María Silva Dreyer ${ }^{\mathrm{e}, \mathrm{b}}$
}

\begin{abstract}
aMatrón. Departamento de Neonatología, Escuela de Obstetricia y Puericultura, Facultad de Medicina Campus San Felipe, Universidad de Valparaíso. Chile

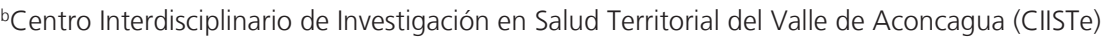

'Alumno de cuarto año, Carrera de Obstetricia y Puericultura, Facultad de Medicina Campus San Felipe, Universidad de Valparaíso

'Matrona. Departamento de Salud Comunitaria, Escuela de Obstetricia y Puericultura, Facultad de Medicina Campus San Felipe, Universidad de Valparaíso. Valparíso, Chile

eSocióloga. Unidad de Investigación Escuela de Obstetricia y Puericultura, Facultad de Medicina Campus San Felipe, Universidad de Valparaíso. Chile
\end{abstract}

Recibido: 26 de noviembre de 2019; Aceptado: 12 de mayo de 2020

\section{¿Qué se sabe del tema que trata este estudio?}

El calostro materno es beneficioso para la salud del recién nacido, por su aporte inmunológico. No obstante, en recién nacidos prematuros, dada su inmadurez general, la lactancia materna se restringe en las primeras horas de vida.

\section{¿Qué aporta este estudio a lo ya conocido?}

Entrega la opinión y experiencia de profesionales de salud de hospitales de alta complejidad neonatal, acerca del suministro precoz, dosificado y protocolizado de calostro a prematuros aún extremos, como medida profiláctica general y preventiva de Enterocolitis necrotizante.

\section{Resumen}

La incidencia de enterocolitis necrotizante $(\mathrm{ECN})$, en Chile es de 0,3 a 2,4 por mil recién nacidos vivos, siendo principalmente afectados los neonatos prematuros, y de 8 a 12 por ciento en prematuros menores a 1.500 gramos. Objetivo: Describir la percepción de profesionales de salud sobre el uso de calostro en recién nacidos prematuros, como factor protector de enterocolitis necrotizante. Sujetos y Método: Estudio cualitativo, mediante entrevista semiestructurada a 18 profesionales de la salud en tres hospitales públicos de la región de Valparaíso. La pauta de entrevista incluyó 3 temas: Co-
Palabras clave:

Enterocolitis;

Parto Prematuro;

Calostro;

Medida Preventiva 
nocimientos, percepción del suministro temprano de calostro y opinión acerca de la extensión de la medida, y 6 subtemas, 2 para cada tema respectivamente: Autopercepción del nivel de conocimiento y fuentes de información; Experiencia: aspectos positivos/eventos adversos y opinión del calostro como factor protector de enterocolitis; aspectos facilitadores u obstaculizadores y opinión acerca de la medida como política nacional. Procesamiento de datos mediante análisis de contenido cualitativo, temático. Resultados: El uso de calostro en prematuros se da de modo protocolizado en dos de las tres unidades de alta complejidad neonatal de la región de Valparaíso. Los participantes opinan positivamente acerca de los resultados preventivos de esta medida. Aun cuando en un tercer establecimiento no se aplique, hay una percepción favorable acerca de su potencial beneficio y su bajo costo de implementación. Se señala, no obstante, que ésta requiere de mayor evidencia y de un protocolo de aplicación. Otras limitantes serían la insuficiente dotación y formación del personal, y la necesidad de adquirir equipamiento e insumos. Conclusiones: Profesionales que han aplicado un protocolo de administración de calostro en neonatos prematuros en la Región de Valparaíso, reportan buenos resultados de salud, y promueven la motivación del equipo hacia esta praxis. Sin embargo, se considera relevante la difusión y discusión de protocolos nacionales e internacionales, así como el desarrollo de investigación local. Dadas las experiencias en curso en Chile, y el debate internacional, se considera oportuno que el tema sea abordado y discutido en la comunidad sanitaria nacional.

\section{Abstract}

In Chile, necrotizing enterocolitis (NEC) mainly affects preterm infants, with an incidence of 0.3 to 2.4 per 1,000 live births, and 8 to $12 \%$ in preterm infants weighing less than 1,500 grams. Objective: To describe health professionals perceptions on the use of human colostrum as a preventive measure against necrotizing enterocolitis in preterm newborns. Subjects and Method: Qualitative study, using 18 semi-structured individual interviews of health professionals in three public hospitals of the Valparaíso Region. The interview included 3 topics: Knowledge, Perception of early colostrum supply and Opinion about the extent of the measure, and 6 subtopics, 2 for each topic respectively: Self-perception of knowledge level and Sources of information; Experience: positive aspects/adverse events and Opinion of colostrum as a protective factor for enterocolitis; Facilitating or hindering aspects and Opinion about the measure as national policy. Data were processed through qualitative content analysis. Results: Two of the three high-complexity neonatal units of the Valparaíso Region have a protocol for administrating colostrum in premature infants. Participants have a positive opinion about the preventive results of this measure. Even in the third hospital where there is no protocol, they have a favorable perception of its potential benefit and its low cost of implementation. However, we observed that this procedure requires more evidence and an application protocol. Other limitations would be the lack of staffing and training and the need for equipment and supplies. Conclusions: Professionals who have applied a colostrum administration protocol in preterm infants in the Valparaíso Region report good health outcomes and promote team motivation towards this practice. However, it is relevant to the dissemination and discussion of national and international protocols, as well as the development of local research. Given the ongoing experiences in Chile and the international debate, we considered appropriate to address and discuss the topic within the national health community.

\section{Keywords:}

Enterocolitis; Premature Birth; Human Colostrum; Prevention Measures

\section{Introducción}

La incidencia de enterocolitis necrotizante (ECN), en Chile es de 0,3 a 2,4 por mil recién nacidos vivos, siendo principalmente afectados los neonatos prematuros, y de 8 a 12 por ciento en prematuros menores a 1.500 gramos $^{1}$. Las orientaciones nacionales para el manejo de prematuros ${ }^{1}$ contemplan, entre otras medidas, el régimen cero de alimentación. No cumplir esta medida podría generar un conjunto de trastornos en el recién nacido, entre ellos, la $\mathrm{ECN}^{2,3}$, dado que su sistema digestivo e inmaduro ${ }^{3}$. Por otro lado, en el recién nacido de bajo peso con factores de riesgo, se debe postergar la alimentación enteral al menos 48 h, y en los que no presentan factores de riesgo, pueden alimentarse desde el primer día ${ }^{1}$. Esta política es coincidente con evidencia y orientaciones nacionales e internacionales de alimentación en prematuros, las que indican que, según la evolución clínica del paciente, podrán ser alimentados vía enteral a partir del primer o segundo día $a^{4-8}$.

En la última década, diversos autores han planteado los beneficios de la alimentación enteral temprana en prematuros, con la finalidad de estimulación 
trófica, aporte inmunológico y prevención de enfermedades gastrointestinales ${ }^{6,9}$; además de tiempo para lograr alimentación enteral completa y favorecer la lactancia materna ${ }^{10,11}$. Diversas revisiones e investigaciones han indagado en el riesgo y beneficio profiláctico de suministro temprano de calostro materno desde las primeras horas de vida, aún en prematuros extremos $^{9-14}$. En éstos, el aporte de calostro se entrega en dosis muy bajas, entre 0,1 y $0,5 \mathrm{ml}$ cada 2 a $4 \mathrm{~h}$. En términos de resultados, se ha reportado la seguridad de su administración en prematuros extremos y enfermos ${ }^{11,14-17}$.

En cuanto a los beneficios, los resultados son divergentes. Ensayos clínicos y revisiones de literatura han establecido una asociación positiva entre el suministro de calostro humano y menor incidencia de morbimortalidad ${ }^{7,17,18}$, y específicamente $\mathrm{ECN}^{12,13,19-24}$. Se discute el volumen y velocidad de suministro ${ }^{25,26}$. No obstante, otros estudios han señalado que la relación no se ha podido establecer, o establecer suficientemen$\mathrm{te}^{10,11,14,16,17,26-33}$. Un ensayo clínico multicéntrico con 622 pacientes enrolados, cuyo protocolo fue publicado el $2015^{33}$, se encuentra actualmente en curso.

Algunos estudios han analizado el impacto positivo de la aplicación de protocolos de nutrición estandarizados sobre la morbimortalidad de los prematu$\operatorname{ros}^{17,22,24}$. Para realizar un análisis exhaustivo de protocolos de alimentación enteral trófica en prematuros se requeriría de un estudio específico. Sin embargo, algunos ejemplos que ilustran posturas que favorecen su uso. La Organización Mundial de la Salud en su informe de acción global sobre nacimientos prematu$\operatorname{ros}^{34}$, promueve la alimentación enteral temprana aún en prematuros extremos, así como también la Asociación Española de Pediatría ${ }^{35,36}$. No obstante, en ese país las prácticas clínicas varían en su régimen de alimentación en prematuros ${ }^{37}$, y hay dudas acerca del beneficio de comenzar la alimentación enteral en las primeras horas $^{38}$. Por otro lado, la Sociedad Iberoamericana de Neonatología recomienda el inicio de estimulación enteral trófica con leche materna lo antes posible luego del nacimiento, aunque recomienda cautela dada la falta de evidencia acerca de los beneficios ${ }^{39}$. En República Dominicana, una guía de práctica clínica para la atención del recién nacido prematuro ${ }^{40}$ favorece el suministro enteral trófico de prematuros, como también lo hace una guía clínica del distrito de salud local Sydney, Australia ${ }^{41}$.

En Chile, se está administrando estímulo mínimo enteral con calostro materno, con el propósito preventivo antes señalado en hospitales de algunas regiones, entre ellas la Metropolitana, Valparaíso, Los Lagos ${ }^{42-45}$. Sin embargo, esta praxis no está extendida, no hay un protocolo de carácter nacional respecto del suministro precoz de calostro en las primeras horas de vida de pre- maturos, y no se encontraron estudios evaluativos de las experiencias nacionales.

Específicamente en la Región de Valparaíso, hay tres establecimientos con unidades de cuidados intensivos neonatales, de los cuales dos aplican la medida de alimentación trófica con calostro materno a prematuros. En uno de ellos se tuvo acceso al protocolo, acorde al cual el calostro de las primeras seis horas se administra en cantidad de $0,5 \mathrm{ml}$ cada tres horas, por ocho veces diaria, vía sonda gástrica o sublingual a los prematuros, incluso extremos estabilizados y sin riesgo de enterocolitis. En un tercer hospital lo anterior no se aplica, dando cuenta de criterios clínicos de manejo diferenciados en una misma región.

Dada la influencia de la experiencia clínica y visión de los profesionales de salud en la discusión, propuesta y ejecución de medidas sanitarias, es de interés conocer su opinión en relación con estas prácticas. Lo anterior como un paso preliminar, que permita establecer un punto de partida para visualizar la predisposición del equipo de salud frente al tema, a la discusión acerca de los riesgos y beneficios de extender la práctica existente en algunos de sus hospitales de alta complejidad, y a la motivación de desarrollar la investigación en torno al tema. Se desprende el objetivo del estudio, que es identificar la opinión del equipo de salud sobre el uso de calostro como factor protector de ECN, en la que se explora.

\section{Sujetos y Método}

\section{Enfoque}

El enfoque metodológico fue cualitativo, de perspectiva fenomenológica ${ }^{46,47}$, dado el objetivo de conocer la percepción del sujeto ${ }^{48}$, en este caso el personal de salud, desde su propia visión y experiencia, la cual influye sobre su opinión, y contribuyen a mantener las praxis o generando los cambios. Este enfoque es de utilidad cuando se quiere comprender la perspectiva de las personas en torno a los fenómenos que los rodean, el significado que les otorgan ${ }^{49,50}$, la conducta desde el propio marco de referencia del participante ${ }^{46}$.

\section{Participantes}

La población estuvo constituida por médicos ginecoobstetras, pediatras, matronas/es, y técnicos superiores en enfermería de unidades de neonatología y ginecoobstetricia, de cada uno de los tres servicios públicos de alta complejidad de la Región de Valparaíso. La muestra correspondió a dieciocho profesionales, lo que permitió cubrir la variedad de las tres áreas disciplinares y tres establecimientos antes señalados $(\text { tabla } 1)^{51}$, y lograr la saturación de los tópicos más relevantes. Los participantes manifestaron su voluntad de colaborar a 
título personal, en tanto informantes con experiencia de más de dos años en la atención de prematuros y sus madres. Para acceder a los participantes, se complementaron diversas técnicas de muestreo cualitativo: en redes o bola de nieve, y por conveniencia ${ }^{49,51}$.

Criterios de exclusión fueron el haber trabajado en el área menos de dos años, o ser reemplazante.

\section{Técnica de recolección de datos}

Se recurrió a la entrevista individual semiestructurada a informantes clave, grabada, guiada por una pauta de entrevista (tabla 2), de aplicación flexible en términos de contenido y secuencia temática de la conversación ${ }^{47,49,51}$.

\section{Análisis}

Para el análisis de los datos se recurrió a la técnica de análisis de contenido cualitativo temático ${ }^{52-56}$, efectuado mediante un proceso de codificación inductivo $^{49,52}$. Es decir, de identificación de ejes temáticos (categorías) y subtemas (subcategorías) que emergen del texto transcrito, con los cuales se codifica cada en- trevista, en un primer nivel de análisis. En un segundo nivel de análisis, se realizó la codificación integrada: se agrupan las categorías y subcategorías, buscando patrones y relaciones entre ellas, considerando los objetivos y dimensiones establecidos para el estudio, así como los temas emergentes no planteados en el diseño inicial del trabajo.

\section{Rigor de investigación}

El equipo de investigación, a inicios del estudio, se sometió a un proceso de identificación de sesgos de los investigadores, capacitación y homologación para la aplicación de las entrevistas, previamente piloteadas. Todas las entrevistas fueron grabadas y transcritas literalmente. Una vez realizadas las primeras, sus resultados fueron discutidos, con el fin de adaptar el instrumento y mejorar su aplicación. El proceso de codificación de todo el texto de cada entrevista fue realizado junto a un proceso de reflexividad acerca de sesgos interpretativos y resultados, por un miembro tesista del equipo, el investigador principal y la asesora metodológica del estudio. Se discutió a la vez el resul-

Tabla 1. Conformación de la muestra

\begin{tabular}{|c|c|c|c|c|}
\hline Profesión y área de competencia & Hospital 1 & Hospital 2 & Hospital 3 & Total \\
\hline $\begin{array}{l}\text { Médico": } \\
\text { Pediatra } \\
\text { Ginecoobstetra }\end{array}$ & $\begin{array}{l}1 \\
1\end{array}$ & $\begin{array}{l}1 \\
1\end{array}$ & 1 & $\begin{array}{l}3 \\
2\end{array}$ \\
\hline $\begin{array}{l}\text { Matrona/matrón²: } \\
\text { Neonatología } \\
\text { Ginecoobstetricia }\end{array}$ & $\begin{array}{l}1 \\
1\end{array}$ & $\begin{array}{l}2 \\
1\end{array}$ & $\begin{array}{l}1 \\
1\end{array}$ & $\begin{array}{l}4 \\
3\end{array}$ \\
\hline $\begin{array}{l}\text { Técnicos en enfermería²: } \\
\text { Neonatología } \\
\text { Ginecoobstetricia }\end{array}$ & $\begin{array}{l}1 \\
1\end{array}$ & $\begin{array}{l}1 \\
1\end{array}$ & $\begin{array}{l}1 \\
1\end{array}$ & $\begin{array}{l}3 \\
3\end{array}$ \\
\hline Total & 6 & 7 & 5 & $\begin{array}{c}18 \\
\text { (13 mujeres y } 5 \text { hombres; } \\
\text { rango de edad: adultos } \\
\text { jóvenes y adultos) }\end{array}$ \\
\hline
\end{tabular}

Tabla 2. Temas Guía de la Pauta de Entrevista

\begin{tabular}{ll}
\hline Tema & Subtemas \\
\hline Conocimientos & $\begin{array}{l}\text { Autopercepción del nivel de conocimiento } \\
\text { Fuentes de información }\end{array}$ \\
Percepción del suministro temprano de calostro & $\begin{array}{l}\text { Experiencia: aspectos positivos y eventos adversos } \\
\text { Opinión del calostro como factor protector de enterocolitis y otras patologías, } \\
\text { actitud frente a su uso profiláctico }\end{array}$ \\
Opinión acerca de la extensión de la medida & $\begin{array}{l}\text { Aspectos facilitadores u obstaculizadores. } \\
\text { Opinión acerca de la medida como política nacional }\end{array}$ \\
\hline
\end{tabular}


tado global y su alcance. Dada la muestra, la transferibilidad de los resultados deberá ser estudiada en otros contextos regionales ${ }^{46,49,56}$.

\section{Aspectos éticos}

El estudio fue aprobado por el Comité de Ética del Servicio de Salud Aconcagua (marzo 2018). Todos los participantes dieron su consentimiento informado a la entrevista, que fue anónima. Los autores no declaran conflictos de interés.

\section{Resultados}

Los resultados que se presentan a continuación incluyen el conocimiento y percepción acerca del suministro precoz de calostro a prematuros, y la opinión acerca de las condiciones de aplicación y la extensión de esta medida a escala nacional.

\section{Percepción del equipo de salud sobre la administración precoz de calostro materno como factor protector de ECN}

La gran mayoría (dieciséis) de los entrevistados posee una percepción positiva de esta medida profiláctica. La principal razón por la cual se cree que la administración de calostro en las primeras horas de vida de los recién nacidos prematuros es positiva, es por la estimulación trófica, y su aporte en inmunoglobulinas. Esta percepción se aplica al conjunto de recién nacidos prematuros, especialmente a los prematuros extremos, e independientemente de su condición. La medida disminuiría el tiempo de hospitalización y con ello el riesgo de infecciones asociadas a la atención en salud. Señalan que diversas investigaciones, como los resultados positivos en sus propios pacientes, que avalan la praxis.

"Pero específicamente para ECN el calostro tiene un rol fundamental. O sea, por los factores antiinfecciosos que tiene, proteinas y células vivas también, y factores que estimulan el crecimiento de la flora intestinal normal. Un probiótico natural, absolutamente, tiene factores oligosacáridos que estimulan la proliferación de la flora bífida, lactobacilos, flora protectora. Por eso el interés del calostro como protector antiinfeccioso, más que con fines nutricionales" (E6P31).

Dos entrevistados, se abstuvieron de una respuesta positiva fehaciente. A juicio de ellos, no se cuenta con evidencia suficiente, aun cuando reconocen los beneficios y componentes protectores generales del calostro. Por ello, no lo aplicarían como profilaxis para prevenir la ECN, pero sí como estimulación enteral, ya que se utilizan volúmenes mínimos de leche materna.
"En base a un estudio chico no lo implementaría. Si estuviesen los recursos haría andar un estudio más grande con más potencia estadística. Y demostrar algún tipo de beneficio ...... hay que sopesar los riesgos versus los beneficios que puede traer, pero con tan poco, es difícil (E10P5)”.

A partir de la experiencia, el total de participantes de los dos hospitales que aplican la medida no cree que ésta tenga contraindicaciones o produzca algún daño en el prematuro, si es adecuadamente dosificada y administrada. Se utiliza como tratamiento inmunológico y se administra como un medicamento dosificado.

\section{Percepción de riesgo de la práctica de administración de gotitas de calostro}

A partir de la experiencia, el total de participantes de los dos hospitales que aplican la medida no cree que ésta tenga contraindicaciones o produzca algún daño en el prematuro, si es adecuadamente dosificada y administrada. Se utiliza como tratamiento inmunológico y se administra como un medicamento dosificado.

"El uso de calostro, lo que yo he visto y lo que se hace acá, es administración de 2 gotas sublinguales, que eso no tendría grandes contraindicaciones en realidad. Prematuros extremos en situaciones críticas conectados a ventilación mecánica, estabilidad hemodinámica que uno no pensaría alimentarlos por vía enteral. Pero la administración de dos gotas de calostro cada tres horas por ocho veces sublingual, no afecta. Y sí tendría un rol protector del punto de vista inmunológico y del punto de vista de disminuir el riesgo de enterocolitis necrotizante" (E2P2).

Los participantes consideraron relevante la técnica aséptica. En los dos hospitales en los cuales se aplica, los entrevistados relatan que se realiza como indicación universal a recién nacidos prematuros, ya que existe un protocolo para ello. Se procura que su extracción sea lo más precoz posible. Los únicos casos en que se posterga esta práctica, es en madres VIH positivo, por el riesgo de transmisión.

\section{Conocimiento e interiorización de la administración de gotitas de calostro}

Respecto al conocimiento acerca del tema, tres distintos profesionales, manifestaron mucho conocimiento acerca del tema.

"Mucho conocimiento, primero por la formación de pregrado y por las capacitaciones que nosotros hemos tenido que hacer acá mismo, ya que hemos tenido capacitaciones de lactancia, con el equipo del Chile Crece" (E1P5). 
Otros trece participantes señalaron tener un conocimiento mediano o insuficiente, y plantean que se requiere mayor evidencia acerca del tema.

"Yo considero que poco, yo creo que todos tenemos poco conocimiento al respecto, se necesita justamente hacer estudios, protocolos grandes. No creo que haya alguien que pueda decir a pie firme "no, yo tengo harto conocimiento al respecto". Porque de partida la ENC es una enfermedad multifactorial... ....Entonces, pensar que el calostro por sí solo influye necesariamente, es un pensamiento muy positivo y está bien. Pero como es tan multifactorial y hoy no se sabe a qué apuntar con mayor fuerza, que el calostro tenga o no que ver con la reducción de la incidencia de ECN, creo que falta muchos estudios, mucha evidencia” (E2P5).

La fuente de información principal fueron cursos de capacitación en su centro asistencial o en otros espacios. Otra fuente han sido protocolos existentes en el Hospital 1 y 2, y publicaciones científicas. Además, la gran mayoría manifestó tener más conocimientos acerca de lactancia materna en general, que específicamente acerca del calostro y su acción profiláctica.

\section{Factores que facilitan u obstaculizan la administra- ción de calostro en recién nacidos}

En cuanto a los recursos para implementar la medida, varios entrevistados señalan que se contaría con los medios económicos para la adquisición de insumos. A la vez, se cuenta con recurso humano competente. Una mayoría (quince) cree que ya se encuentran capacitados y con buena disposición para la ejecución de esta práctica, sobre todo en las unidades de neonatología. Adicionalmente, los establecimientos cuentan con lactario e insumos básicos, a los que se podría recurrir.

"Yo creo que existe la infraestructura, que existe el recurso humano. Hay que ver qué necesitamos del punto de vista de insumos, pero en términos generales yo creo que sí. Acá tenemos un lactario donde la mamá se extrae leche donde se almacena la leche, que pueden utilizar por supuesto para la extracción segura de calostro, las condiciones están, más menos incómodas, pero están" (E2P12).

Sin embargo, también se plantean dificultades. Entre ellas, que no todos los hospitales el país poseen las mismas facilidades y recursos, y no siempre la disposición del personal es positiva en el conjunto del equipo. Algunas de las dificultades mencionadas son el acceso a la madre para la extracción precoz de calostro, ya que ésta puede encontrarse en una unidad de tratamiento intermedio, o unidad de cuidado intensivo. $\mathrm{O}$ bien el apego de algunos profesionales a la indicación de régimen cero en recién nacidos prematuros. Finalmente, se aduce la falta de información sobre el calostro y sus beneficios, en particular en establecimientos que aún no implementan esta praxis.

"Yo creo que existe la infraestructura, que existe el recurso humano. Hay que ver qué necesitamos del punto de vista de insumos, pero en términos generales yo creo que sí. Acá tenemos un lactario donde la mamá se extrae leche donde se almacena la leche, que pueden utilizar por supuesto para la extracción segura de calostro, las condiciones están, más menos incómodas, pero están" (E2P12).

\section{Implementación de la praxis de administración precoz de calostro en prematuros, como política nacional}

La mayoría de los entrevistados, principalmente los que se desempeñan en los dos hospitales que aplican la medida, afirma que la profilaxis de gotitas de calostro debería establecerse como una política a nivel nacional. Lo anterior por los beneficios observados en recién nacidos prematuros, y la posibilidad de implementación de bajo costo. Algunos profesionales afirman que esta práctica ya es efectuada en algunas ciudades del país. Si en otras ciudades no se ha efectuado, se piensa que podría deberse a la falta de información.

"Tiene un costo en cuanto a recursos que es muy bajo. El efecto salud que tiene sobre el paciente, la enterocolitis necrotizante, comparando el efecto-costo, es muy amplia la ventaja" (E7P9).

Algunos, sin embargo, en particular participantes del hospital en el cual no se aplica la medida, creen que no debiera aplicarse a escala nacional. Aun cuando su percepción general es positiva, opinan que primero debiesen desarrollarse investigaciones y evaluarse resultados en centros centinela, con el fin de comprobar los beneficios en recién nacidos sometidos a este tratamiento preventivo. A la vez, se debiese sensibilizar y capacitar al recurso humano para una implementación exitosa. En caso de realizarse, debiera contarse con una guía clínica nacional, y protocolos de aplicación precOZ.

"No sé si a nivel país todavía. Me parece bien que algunos centros la estén adoptando. Y quizás reunir casos y ver cómo les está yendo, los resultados preliminares. $Y$ si se ven buenos resultados, copiar los buenos ejemplos y que se adopte a nivel país. Pero claro, a veces estos centros centinelas que son los que han adoptado esta práctica, sería bueno que reporten estos resultados y demostrar si funciona" (E10P7). 


\section{Discusión}

En este estudio, los profesionales de salud entrevistados tienen una percepción positiva de la administración precoz de calostro en recién nacidos prematuros, por sus propiedades inmunológicas, de estímulo trófico, y por considerarse un método de bajo riesgo. Esta actitud positiva es coincidente con los resultados de investigaciones realizadas en diversos países ${ }^{9-24}$ y recomendaciones de algunos servicios de neonatología en Chile $^{42-45}$.

Dada la situación favorable descrita en centros hospitalarios de alta complejidad de otras regiones del país, surge la pregunta por la eventual generalización de esta medida. Del presente estudio, se desprenden algunas de las posibles respuestas. Los principales factores obstaculizadores se refieren a aspectos índole científico-clínico, institucional y organizacional.

Respecto al primero, varios participantes plantean que la evidencia científica es aún insuficiente, lo que refleja la discusión científica internacional. Acorde a ésta, hay estudios que favorecen la praxis de suministro enteral trófico con calostro de la madre de las primeras horas de vida, mientras otros discuten su utilidad o nivel de evidencia para la prevención de morbimortalidad neonatal, y específicamente, de $\mathrm{ECN}^{10,11,14,16,17,26-33}$. Otra razón aducida por algunos participantes es el desconocimiento del conjunto de experiencias desarrolladas en Chile. Cabe señalar que, en la búsqueda de antecedentes para el diseño del presente estudio, no se encontraron publicaciones con los resultados de las experiencias en curso en el país.

En cuanto a la segunda limitante, de índole institucional, se plantea la inexistencia de una política ministerial de alcance nacional, con su respectiva guía clínica y protocolo dirigido específicamente a la administración enteral precoz de calostro materno de las primeras horas como medida profiláctica. También se teme a los eventuales riesgos asociados a la aplicación de esta medida, y las limitantes de un contexto de arraigo profesional a la indicación de régimen cero en los recién nacidos prematuros extremos.

En el plano organizacional, el problema central planteado por los participantes del estudio es la insuficiente dotación de personal. En un contexto de sobrecarga laboral, la aplicación de nuevas técnicas, cambio de hábitos y nuevas formas de organización del trabajo, podría verse enfrentada a cierta pasividad o resistencia entre los profesionales de salud. Por tanto, sería fundamental la sensibilización e información al equipo profesional. A la vez, es necesaria la coordinación entre los servicios de maternidad y neonatología. Otra difi- cultad es que no todos los establecimientos con unidades de cuidados intensivos neonatológicos cuentan con los mismos recursos, y aun cuando la medida no sería de alto costo, sí tendría que disponer de algunos recursos de implementación. La objetivación mediante estudio de la relación costo-beneficio que implicaría la implementación de esta práctica, podría ser un aporte al debate de una eventual política nacional.

A modo de síntesis de los antecedentes revisados y los resultados del presente estudio, se plantea la pertinencia y oportunidad de abordar y discutir el tema en un futuro próximo. Los resultados permiten suponer la existencia de un terreno subjetivo fértil para la ampliación de esta práctica a escala regional, y eventualmente nacional, en el caso de que, en un futuro, se adopte como política nacional. Sin embargo, es necesario catastrar y difundir las experiencias existentes, y discutir el tema en eventos de la comunidad científica. Así como también desarrollar estudios tanto retrospectivos de resultado y proceso de estas experiencias, como estudios prospectivos, acerca de la seguridad e impacto de la medida.

La limitación central del presente trabajo es la muestra restringida a una sola región (Valparaíso). En un futuro estudio, de mayor cobertura, sería también importante la participación en la muestra de personal de nivel directivo de los establecimientos, no incluidos en este trabajo.

\section{Responsabilidades Éticas}

Protección de personas y animales: Los autores declaran que los procedimientos seguidos se conformaron a las normas éticas del comité de experimentación humana responsable y de acuerdo con la Asociación Médica Mundial y la Declaración de Helsinki.

Confidencialidad de los datos: Los autores declaran que han seguido los protocolos de su centro de trabajo sobre la publicación de datos de pacientes.

Derecho a la privacidad y consentimiento informado: Los autores han obtenido el consentimiento informado de los pacientes y/o sujetos referidos en el artículo. Este documento obra en poder del autor de correspondencia.

\section{Conflicto de intereses}

Los autores declaran no tener conflicto de intereses. 


\section{Referencias}

1. Mena P, Mülhausen G, Novoa J, Vivanco G. Guía Nacional de Neonatología. Ministerio de Salud. Guía Nacional de Neonatología. Santiago, Chile 2005.

2. Bell MJ, Ternberg JL, Feiguin RD, et al. Neonatal necrotizing enterocolitis. Therapeutic decision based upon clinical staging. An Surg. 1978:1-7.

3. Méndez A, Bancalari A. Ernst I. Enterocolitis necrotizante. Experiencia de 15 años. Rev. Chil. Pediatr. 2000;71(5):390-7.

4. Tapia JL y González A. Recién nacido menor de 1.500 gramos al nacer: enfoque general. En: Tapia JL y González A. Neonatología. Tercera edición. Capítulo 7. Editorial Mediterráneo. Santiago, Buenos Aires. 2008:85-95.

5. José María Ceriani JM, Mariani G, Lupo EA, Jenik A. Neonatología práctica. Cuarta edición. Editorial Médica Panamericana. Argentina. 2011.

6. Cloherty J, Eichenwald E, Hansen A, Stark A. Manual de cuidados neonatales. Ediciones Journal. Libros para profesional de la salud Editorial: Lippincott William and Wilkins. Séptima edición. Filadelfia. 2012. ISBN:9788415419570.

7. Lawn JE, Davidge R, Paul VK, Von Xylander S, De Graft J, Costello A, Kinney MV, Segre J, Molyneux L. Born too soon; care for the preterm baby. Reproductive Health 2013, 10 (Suppl 1):S5.

8. García-López R. Composición e inmunología de la leche humana. Acta Pediátrica de México. 2011;32(4):223-30.

9. Rodríguez NA, Meier PP, Groer MW, Zeller JM. Oropharyngeal administration of colostrum to extremely low birth weight infants: theoretical perspectives. Journal of Perinatology: Official Journal of the California Perinatal Association. 2009;29(1):1-7. Recuperado el 14 de agosto de 2017 de: http://doi.org/10.1038/ jp.2008.130.

10. Sadeghirad B, Morgan R, Zeraatkar D, Zea A, Couban R, Johnston B, Florez I. Human and Bovine Colostrum for Prevention of Necrotizing Enterocolitis: A Meta-analysis. Pediatrics August 2018;142(2):e20180767; DOI: https://doi. org/10.1542/peds.2018-0767.

11. Snyder R, Herdt A, Mejías-Cepeda N, Ladino J, Crowley Kathryn, Levy Philip. Early provision of oropharyngeal colostrum leads to sustained breast milk feedings in preterm infants. Pediatrics and Neonatology. 2017;58:534-40.

12. Talavera M, Bixler G, Cozzi C, Dail J, Miller R, McClead R, Reber K. Quality Improvement Initiative to Reduce the Necrotizing Enterocolitis Rate in Premature Infants. PEDIATRICS 2016;137(5):e 20151119.
13. Good M, Sodhi Ch P, Hackam DJ Evidence based feeding strategies before and after the development of necrotizing enterocolitis. Expert Rev Clin Immunol. 2014; 10(7):875-84. doi:10.1586/174466 6X.2014.913481.

14. Nasuf AWA, Ojha S, Dorling J. Oropharyngeal colostrum in preventing mortality and morbidity in preterm infants (Review). Cochrane Database of Systematic Reviews 2018, Issue 9. Art. No.: CD011921. DOI: 10.1002/14651858. CD011921.pub2.

15. Rodríguez NA, Meier P, Groer M, Zeller J, Engstrom J, Fogg L. Oropharyngeal administration of own mother's colostrum to extremely low birth weight infants. Adv Neonatal Care. 2010;10(4):206-12.

16. Panchal H, Athalye-Jape G, Patole S. Oropharyngeal Colostrum for Preterm Infants: A Systematic Review and Meta-Analysis. Advances in Nutrition. 2019;10(6):1152-62. https://doi. org/10.1093/advances/nmz033.

17. McCallie KR, Lee HC, Mayer O, Cohen RS, Hintz SR, Rhine WD. Improved outcomes with a standardized feeding protocol for very low birth weight infants. Journal of Perinatology. 2011;31:S61-S67.

18. Martín E, Jiménez MV, Peña M, Serrano L, Kajarabille N, Díaz J, Ochoa JJ, Maldonado J. Efectos de la administración de calostro orofaríngeo en recién nacidos prematuros sobre los niveles de inmunoglobulina. A. Nutr Hosp 2016;33:232-8.

19. Cristófalo E, Schanler R, Blanco C, et al. Randomized trial of exclusive human milk versus preterm formula diets in extremely premature infants. The Journal of Pediatrics 2013;163(6):1592-5.

20. Sisk PM, Lovelady CA, Dillard RG, Gruber KJ, O'Shea TM. Early human milk feeding is associated with a lowe risk of necrotizing enterocolitis in very low birt weight infants. Journal of Perinatology. 2007;27:428-33.

21. Underwood M. Human milk for the premature infant. Pediatr Clin North A. February 2013;60(1):189-207.

22. Gephart SM, Hanson C, Wetzel CM, Fleiner M, Umberger E, Laura Martin L, Rao S, Agrawal A, Marin T, Kirmani K, Megan Quinn M, Quinn J, Dudding KM, Clay T, Sauberan J, Eskenazi Y, Porter C, Msowoya AL, Wyles C, Avenado-Ruiz M, Vo S, Reber KM, Duchon J. NEC-zero recommendations from scoping review of evidence to prevent and foster timely recognition of necrotizing enterocolitis. Maternal Health, Neonatology, and Perinatology. 2017;3:23. DOI 10.1186/ s40748-017-0062-0.

23. Lee J, Kim HS, Young Hwa Jung, Ka Young Choi, Seung Han Shin, Ee-
Kyung Kim, Jung-Hwan Choi. (2015). Oropharyngeal Colostrum Administration in Extremely Premature Infants: An RCT. Pediatrics. 2015;135(2):357-66.

24. Jin Y, Duan Y, Deng X, Lin J. Prevention of necrotizing enterocolitis in premature infants-an updated review. World J Clin Pediatr. 2019;8(2):23-32. ISSN 2219-2808 (online).

25. Oddie SJ, Young L, McGuire W. Slow advancement of enteral feed volumes to prevent necrotising enterocolitis in very low birth weight infants. Cochrane Database of Systematic Reviews 2017, Issue 8. Art. No.: CD001241. DOI: 10.1002/14651858.CD001241.pub7.

26. Mena P, Kaitan J, Zavala A. Enterocolitis necrotizante. En: Tapia JL y González A. Neonatología $3^{a}$ edición. Editorial Mediterráneo. Santiago, Chile 2008;59:683-91.

27. Garg BD, Balasubramanian H, Kabra NS, Bansal A. Effect of oropharyngeal colostrum therapy in the prevention of necrotising enterocolitis among very low birthweight neonates: A meta-analysis of randomised controlled trials. J Hum Nutr Diet. 2018;31:612-24 https://doi. org/10.1111/jhn.12585.

28. Bührer C, Fischer HS, Wellmann S. Nutritional interventions to reduce rates of infection, necrotizing enterocolitis and mortality in very preterm infants. Pediatric Research (2020)87:371-7; https://doi.org/10.1038/s41390-019-06302.

29. Meinzen-Derr J, Poindexter B, Wrage L, Morrow AL, Stoll B, Donovan EF. Role of human milk in extremely low birth weight infants' risk of necrotizing enterocolitis or death. Journal of Perinatology. 2009;29:57-62.

30. Demestre X, Raspall F. Protocolos de Neonatología. (2da ed). Barcelona, España: Asociación Española de Pediatría. 2008.

31. Foster JP, Seth R, Cole MJ. Oral inmmunoglobulin for preventing necrotizing entercolitis in preterm and low birth weight neonates (Review). Cochrane Database of Systematics Reviews. 2016 Apr.4; 4:CD001816. doi: 10.1002/14651858.CD001816.pub3.

32. Meinzen-Derr J, Poindexter B, Wrage L, Morrow Al, Stoll B, Donovan EF and the National Institute of Child Health and Human Development Neonatal Research Network. Role of human milk in extremely low birth weight infants'risk of necrotizing enterocolitis and death. J Perinatol. January 2009;29(1):57-62.

33. Rodríguez NA, Maximo Vento M, Claud E, Wang C, Caplan M. Oropharyngeal administration of mother's colostrum, health outcomes of premature infants: study protocol for a randomized 
controlled trial. Trials (2015) 16:453 DOI 10.1186/s13063-015-0969-6.

34. March of Dimes, PMNCH, Save the Children, WHO. Born Too Soon: The Global action report on preterm Birth. eds CP Howson, MV Kinney, JE Lawn. World Health Organization. Geneva, 2012.

35. Bustos G. Alimentación enteral del recién nacido pretérmino. Protocolos de la Asociación Española de Pediatría, Sociedad Española de Neonatología. En revisión. $2^{\text {a }}$ edición. 2008. Capítulo 7. Pgs 58 a 67. Recuperado 22.03 .2020 de https://www.aeped.es/sites/default/files/ documentos/7_1.pdf

36. Demestre X, Raspall F. Protocolos de Neonatología (en revisión), Asociación Española de Pediatría. Cap 42: recuperado el 20 de marzo de 2020 de: https://www.aeped.es/sites/default/files/ documentos/42.pdf

37. Alonso-Díaz C, Utrera-Torres I, AlbaRomero C, Flores-Antóna B, LópezMaestro M, David Lora-Pablos D, PallásAlonso CR. Prácticas de alimentación con leche materna en recién nacidos menores de $1.500 \mathrm{~g}$ o de menos de 32 semanas. An Pediatr (Barc) 2016;85(1):26-33.

38. Sánchez-Tamayo T, María G. Espinosa MG, Moreno MC, Fernández V, Triano JV, Tapia E, Salguero E. Nueva guía de práctica clínica sobre nutrición enteral del recién nacido de muy bajo peso al nacimiento; primera parte. Nutr Hosp. 2014;30(2):321-8

39. Rogido M, Golombek SG, Baquero H, Borbonet D, Goldsmit G, Lemus L, Lima V, Morgues M, Natta D, Osiovich H, Oviedo AN, Pérez JM, Rodríguez S, Sola A, Zambosco G, Tercer Grupo de Consenso Clínico SIBEN, Berseth CL, Thureen P, Higuera J. Sociedad Iberoamericana de Neonatología. Tercer
Consenso Clínico SIBEN: Nutrición del recién nacido enfermo. 2009.

40. Ministerio de Salud Pública. Guía de práctica clínica para la atención del recién nacido prematuro. Santo Domingo, República Dominicana. 2008. ISBN: 9789945-591-66-8.

41. Guideline. Women and babies: enteral nutrition for the preterm infant. Document No: RPAH_GL2014_042. NSW Government. Health Sydney Local Health District. Recuperado 5.03.2020 de: https://www.slhd.nsw.gov.au/rpa/ neonatal\%5Ccontent/pdf/guidelines/ RPAH_Feeding_GL2014_042.pdf

42. Mühlhausen G, González A. (editores) Guía de Práctica Clínica. Manual de Neonatología. Hospital San José. Santiago. 2016 (337-342). Recuperado 13/06/2019 de: http://www.neopuertomontt.com/ guiasneo/Guias_San_Jose/Guias_ SanJose_2016.htm

43. Abusleme I. Cuenta Pública, trabajando por el nuevo hospital. Gestión 2018. Hospital Clínico Félix Bulnes. Recuperado el 11 de marzo de 2020 de: https:// felixbulnes.cl/transparencia/Cuenta_ Publica_2018.pdf

44. Hospital Dr. Gustavo Fricke. Hospital Dr. Gustavo Fricke expuso sobre exitosa experiencia de extracción precoz de gotitas de calostro. 2017. Recuperado 13/06/2019 de: http://www.hospitalfricke. $\mathrm{cl} / \mathrm{p}=7213$

45. Hospital Puerto Montt, Servicio de Neonatología. Protocolo alimentación enteral del prematuro menor a 32 semanas o menor a $1.500 \mathrm{~g}$. Recuperado 13/06/2019 de: http://www. neopuertomontt.com/Protocolos/ Protocolo\%20Alimentacion\%20 Enteral\%20Prematuro.pdf.

46. Krause M. La investigación cualitativa-Un campo de posibilidades y desafíos. Revista Temas de Educación. 1995;7:19-39. ISSN 0716-7423.

47. Taylor SJ y Bogdan R. Introducción a los métodos cualitativos de investigación. La búsqueda de significados. Editorial Paidós. España 1987.

48. Galeano ME. Estrategias de investigación social cualitativa. El giro en la mirada. La carreta editores E.U. Medellín, 2004.

49. Hernández R, Fernández C, Baptista P. Metodología de la Investigación. Quinta edición. Mc Graw Hill. 2010.

50. Arteaga O. Investigación en Salud y Métodos Cualitativos. Ciencia y Trabajo. 2006;8(21):151-3

51. Flick U. El diseño de investigación cualitativa. Colección: investigación cualitativa. Ediciones Morata S.L. Madrid 2015.

52. Braun V, Clarke V. Using thematic analysis in psychology. Qualitative Research in Psychology. 2006;3(2):77101. ISSN 1478-0887 matic analysis in psychology.

53. Andreu Jaime (2002). Las técnicas de análisis de contenido: una revisión actualizada. Sevilla: Fundación Centro de Estudios Andaluces. S200103. Recuperado 23.03.2020 de: https:// www.centrodeestudiosandaluces.es/ publicaciones/tecnicas-de-analisis-decontenido-una-revision-actualizada

54. Díaz C. Investigación cualitativa y análisis de contenido temático. Orientación intelectual de revista Universum. Rev. gen. inf. doc. 2018;28(1):119-142 119.

55. Navarro P, Díaz C. El análisis de contenido. En Delgado JM y Gutiérrez J (editores). Métodos y técnicas cualitativas de investigación en ciencias sociales. capítulo 7:177-224. Editorial Síntesis SA. Madrid 1995. 